\section{Tissue Rotation Chamber for Electrophysiology, Microinjection, and Microdissection}

\section{BioTechniques 33:38-42 (July 2002)}

To generate complete 3-D maps of extracellular ion current patterns around insect ovarioles without removing the sample and chamber from the recording equipment, it was necessary to design a chamber permitting complete rotation of the tissue. While suction pipets have been used in previous studies to orient the tissue (1), their use can significantly disturb extracellular ionic gradients if an incomplete seal is obtained. Additionally, it was necessary to introduce a porous surface beneath the ovariole to cushion it and to permit free passage of ions so that current patterns would not be disturbed. Damage and stress to the ovariole during rotation and recording must be negligible, so a mechanism to lift the tissue off the surface was required. The recording chamber described here achieved these goals and may benefit studies of other tissues involving electrophysiology, microinjection, or microdissection where precise orientation of the sample is required.

Figure 1A illustrates the completed chamber, which begins with a $35-\mathrm{mm}$ plastic Petri dish modified as outlined in the figure legend. The rotating shaft and the roller can be manipulated from outside the dish, minimizing disturbance. To begin construction, a hot razor blade is used to remove a $25-\mathrm{mm}$ section of the Petri dish wall. A piece of glass microscope slide the height of the dish (10-11 mm) is cut and siliconed to the opening, permitting sideview observation of the sample via a long focal distance video camera.

After a hole $10-15 \mathrm{~mm}$ in diameter is cut in the dish floor using a Dremel tool, a 22-mm diameter glass coverslip is siliconed in place over the opening to im prove optical clarity, a common practice. A $10-\mathrm{mm}$ diameter plastic ring, made from a $1-\mathrm{mm}$ slice of a $1.5-\mathrm{mL}$ microcentrifuge tube, is then siliconed to the coverslip. This creates a shallow reservoir to be filled with agarose, providing a permeable, transparent tissue support.
Construction of the mechanism for tissue orientation begins by making a hole in the side of the dish, approximately $2 \mathrm{~mm}$ above the dish floor, with a hot 16-gauge syringe needle. A 22gauge syringe needle is prepared by sealing the plastic base with silicone to prevent media leakage. The needle is inserted through a short piece (approximately $3 \mathrm{~mm}$ ) of polyethylene tubing with an inner diameter that gives a snug fit, permitting easy rotation of the metal shaft without leaking media. The tubing and needle are then fitted into the hole in the Petri dish and the tubing is glued in place, oriented so that the syringe needle is parallel to the glass side and pointed toward the center of the plastic ring. Additionally, the needle tip is positioned so it is just below the top of the plastic ring (see Figure 1B). Proper positioning of the syringe is necessary to enable the roller, added subsequently, to adjust the tissue's height relative to the agarose support. The plastic base of the syringe needle can be grasped to rotate the needle and the specimen.

Next, a $15-\mathrm{mm}$ length of thin $(0.127$ $\mathrm{mm}$ ) tungsten wire (Alfa Products, Danvers, MA, USA) is bent to form a clip to grasp the tissue (Figure 1C) and inserted into the syringe needle tip. The wire must be long enough that the clip can be placed inside the plastic ring. A few slight bends are made in the wire to anchor it firmly in the syringe needle so

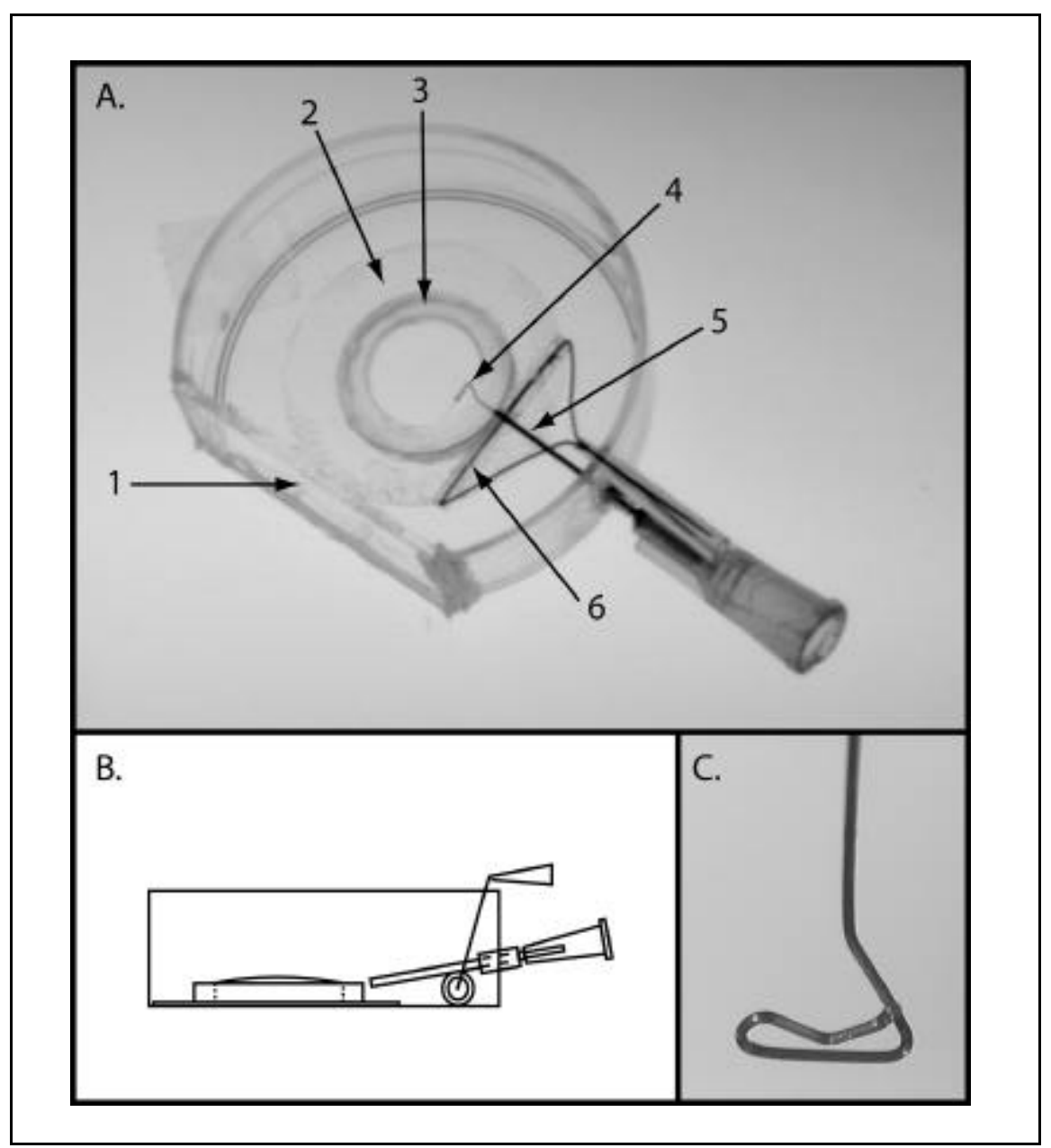

Figure 1. Details of chamber construction. (A) The chamber consists of a glass wall (1) allowing observation via a side-mounted camera, a glass coverslip (2) underneath a plastic ring (3) containing an agarose layer on which the sample rests, a tissue-securing clip (4) inserted into a syringe needle shaft (5), enabling rotation of clip and sample, and a roller (6) that adjusts the height of the clip relative to the agarose floor. (B) The syringe needle tip is oriented so that it rests below the height of the plastic ring, as shown in this side-view diagram. (C) The wire used to hold the sample is bent to form a simple clip. Other tissues may require modification of clip dimensions. 
that both components rotate as a single unit. Since the wire is not glued in place, it can be easily removed for replacement or modification. While it is advantageous to ensure the clip lies along the centerline of the needle to minimize deviation from the center during rotation, complete accuracy is unnecessary because height adjustments can be made using the roller once the sample is in place. The clip is used to secure a region of the tissue that will not be required during experimentation. In our case, we use the pedicel at the posterior end of the ovariole, since it lies along the center of the ovariole and is not physiologically coupled to the rest of the tissue via gap junctions.

Next, a roller constructed from a 15$\mathrm{mm}$ length of glass capillary tubing is

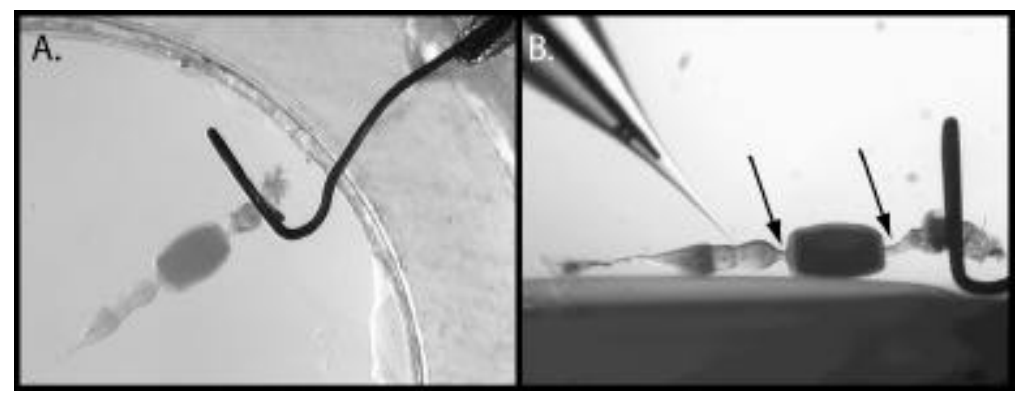

Figure 2. The recording chamber in use. (A) An ovariole from the insect $R$. prolixus held in preparation for an extracellular ion-selective probe study. The tungsten clip secures the posterior end of the ovariole by the expendable pedicel (upper right). The anterior end of the tissue is loosely held in place by plunging the terminal filament into the agarose. (B) A side-mounted camera permits the accurate placement of the probe along the ovariole midline and illustrates that although different regions of the ovariole vary in diameter, the height of the posterior end of the tissue can be adjusted to eliminate stress on the tissue, especially at the thin connective stalks joining larger oocytes (arrows).

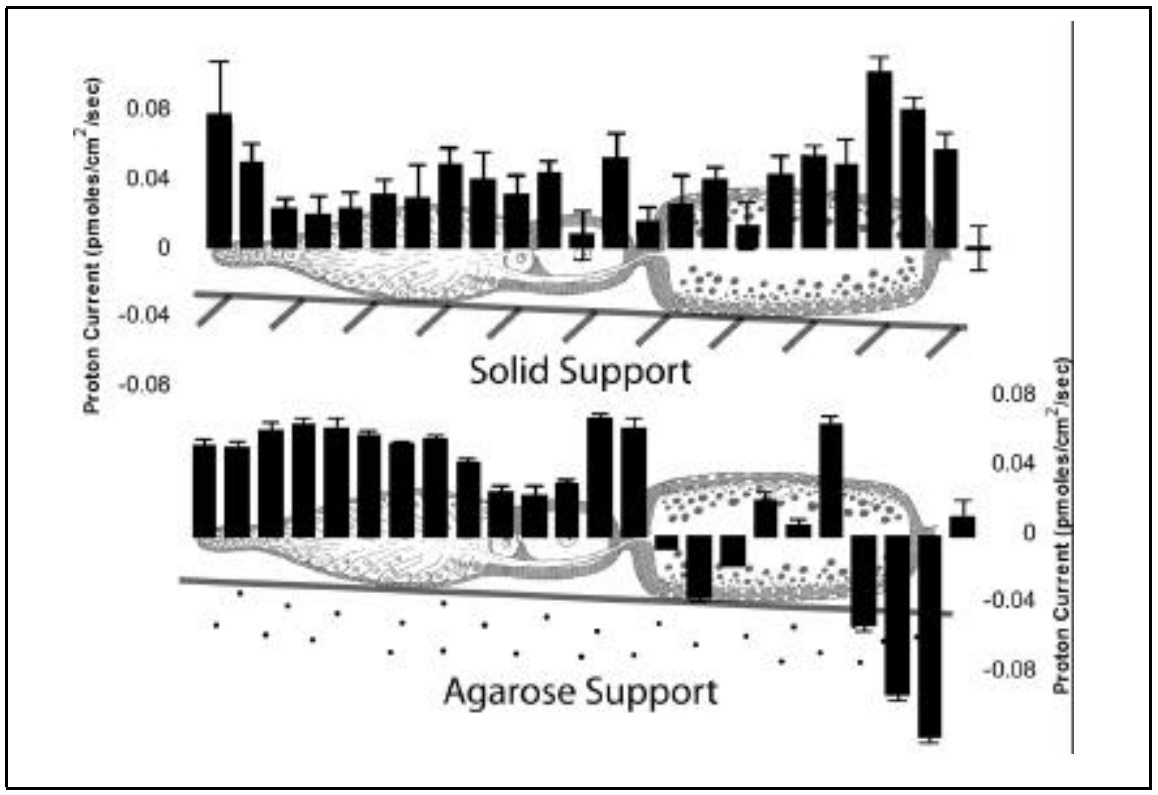

Figure 3. Effects of the agarose layer on extracellular proton currents. The vertical components (black bars) of 3-D proton efflux current present measurements from scans of single ovarioles of similar developmental stage but on different substrates. Measurements were taken along the lateral midline of each ovariole (base of bars). Each bar represents the mean of five 1-s measurements \pm SD. Proton currents measured at all positions from ovarioles resting on a solid plastic surface (top graph) travel away from the dish floor (positive values), implying that the solid barrier underneath may affect them. Currents measured from ovarioles resting on an agarose layer (bottom graph) revealed that at a number of positions currents traveled toward the floor, suggesting that ion traffic around the entire ovariole is unimpeded. placed under the syringe needle. The outer diameter of the tube must be greater than the height of the syringe needle tip off the dish floor so that it can elevate the needle. We use TW1504 capillary tubing with a $1.5-\mathrm{mm}$ o.d. (World Precision Instruments, Sarasota, FL, USA). A thin wire $(0.25 \mathrm{~mm}$ tungsten; Alfa Products) is inserted through the tube, bent at both ends, and extended back to rest on the rim of the Petri dish above the syringe needle, where it serves as a handle. To create a grip, the small end of a micropipettor tip is trimmed to cover the bare wire (Figure 1A). As the roller is pushed towards the plastic ring, it raises the needle tip and the wire clip, lifting the sample off the agarose bed.

Finally, a $0.5 \%$ agarose solution is poured into the plastic ring. This provides an optically clear, porous surface that allows for diffusion of ions and can be replaced as needed. If the agarose is reused, then the chamber should first be sterilized with $70 \%$ ethanol and thoroughly rinsed in the experimental media (physiological saline, etc.). Grooves or extra support can be carved into or added to the agarose layer as required, and a backstop can be added to brace the tissue during microinjection procedures.

Recording chambers constructed in this way have been used successfully in our laboratory to orient ovarioles from the insect Rhodnius prolixus during extracellular ion current analysis using an ion-selective vibrating probe (Applicable Electronics, Sandwich, MA, USA, and Science Wares, Falmouth, MA, USA), without removing them from the recording equipment. Figure 2 shows images of an ovariole being scanned. A single chamber can be used for ovarioles at every developmental stage, even though the vitellogenic oocytes vary greatly in size, without creating undue stress on the ovariole (Figure 2). With practice, the tissue can be rotated in less than $20 \mathrm{~s}$.

The agarose layer has a significant impact in facilitating the measurement of normal extracellular ion currents. Proton efflux currents traveling toward the dish floor were very rarely measured using ovarioles resting on a solid plastic surface. This raised concern that ions traveling toward the floor may be impeded, yielding a distorted 3-D cur- 
rent pattern. Using a porous agarose layer to support the sample, we frequently measure proton currents traveling towards the dish floor, suggesting that this provides a more physiological environment in which ions are free to move from all surfaces of the ovariole unimpeded (Figure 3).

The recording chamber provides a physiological environment and specimen manipulation and orientation possibilities that are well suited to extracellular electrophysiological investigation of a variety of tissues and can be adapted to other studies requiring precise sample orientation.

\section{REFERENCE}

1.Diehl-Jones, W.L. and E. Huebner. 1992. Spatial and temporal transcellular current patterns during oogenesis. Dev. Biol. 153:302311.

Canadian NSERC research and equipment grants to E.H. are gratefully acknowledged. We thank C. Kelly for editorial com ments. Address correspondence to Dr. Chris S. Bjornsson, Department of Zoology, University of Manitoba, Ft. Garry Campus, Winnipeg, MB, Canada R3T 2N2. e-mail: chrisbjornsson@hotmail.com

Received 26 September 2001; accepted 21 March 2002.

\section{Chris S. Bjornsson and Erwin Huebner University of Manitoba Winnipeg, MB, Canada}

For reprints of this or any other article, contact Reprints@BioTechniques.com
Storage of Various Cell Lines at $-\mathbf{7 0}^{\circ} \mathrm{C}$ or $-\mathbf{8 0}^{\circ} \mathrm{C}$ in Multi-Well Plates While Attached to the Substratum

\section{BioTechniques 33:42-46 (July 2002)}

Cryopreservation of higher eukaryotic cell lines is generally accom plished by addition of a cryopreservative (generally glycerol or DMSO) to the cell suspension. This combined with controlled cooling and thawing rates is thought to prevent dehydration and lethal injury during the freezing and thawing stages $(1-3,10,11,13,14)$. When screening panels of clones derived from mammalian cells, it is useful to store the panel in a frozen state during screening to prevent contamination, to eliminate constant maintenance, or to facilitate absences from the laboratory. This report describes a simple method of freezing cells while still attached to the substratum of a multi-well plate. There have been previous reports describing the frozen storage of hybridoma cell lines in 96-well plates $(8,21)$, one of them while cells were still attached to the substratum. Our experiments differ from the previous reports in five major ways. (i) We have simplified the previously reported methods by eliminating time-consum ing media addition steps. (ii) We used a standard cryopreservative consisting of $10 \%$ DMSO, 20\% FBS, and 70\% RPMI 1640 (Invitrogen, Carlsbad, CA, USA) for storage at $-70^{\circ} \mathrm{C}$. (iii) $\mathrm{We}$ avoided use of feeder cells. (iv) We carried out experiments to assess recovery at four different DMSO:FBS ratios. (v) Importantly, our observations with six commonly used mammalian cell lines, including human breast cancer, keratinocytes, and rhabdomyosarcoma, show that the ability to store cells in a frozen state while attached to a substratum is not unique to hybridomas, making this technique widely applicable in modern molecular biology laboratories.

We demonstrated effective storage of six mammalian cell lines for up to one month in a DMSO-containing storage solution at $-70^{\circ} \mathrm{C}$ or three months at $-80^{\circ} \mathrm{C}$, while still attached to the substratum of a multi-well plate (Table 1). The technique bypasses the detachment and centrifugation steps required by the standard storage protocols and offers a significant savings of time when cryopreserving panels of single-cell clones.

Cells were seeded into multi-well dishes (Falcon ${ }^{\circledR}$ low-evaporation, flatbottom tissue culture plates; Fisher Scientific, Pittsburgh, PA, USA) and grown in RPMI $1640+10 \%$ vol $/ \mathrm{vol}$ NUSERUM $^{\mathrm{TM}}$ IV (Becton Dickinson Labware, Franklin Lakes, NJ, USA) or 9\% FBS (Sigma, St. Louis, MO, USA), at indicated confluencies for $24-30 \mathrm{~h}$ at $37^{\circ} \mathrm{C}$ in a $5 \% \mathrm{CO}_{2}$ incubator. The medium was then aspirated, and cells were overlaid with a warm cell freezing solution [10\% vol/vol DMSO (Sigma), 20\% FBS (Invitrogen), and 70\% RPMI 1640]. Either $1 \mathrm{~mL} /$ well or $100 \mu \mathrm{L} /$ well of freezing solution was applied to 12well or 96-well plates, respectively. Each plate was then either sealed in a hybridization bag (Invitrogen) or double-bagged with Zip-Loc ${ }^{\circledR}$ freezer bags to prevent water condensation and im mediately placed into a Styrofoam box $(18 \times 18 \times 9 \mathrm{~cm}$, with wall thickness of $5 \mathrm{~cm}$ ) packed with paper towels. A lid of $2.5 \mathrm{~cm}$ thickness was then taped on, and this "freezing box" was placed in the ultralow freezer $\left(-70^{\circ} \mathrm{C}\right.$ or $\left.-80^{\circ} \mathrm{C}\right)$; after $18-24 \mathrm{~h}$, the plate was removed from the box and stored in ultralow freezer until thawing.

One month or three months after freezing, plates were removed from hybridization bags and placed in a $37^{\circ} \mathrm{C}+5 \% \mathrm{CO}_{2}$ incubator for $20 \mathrm{~min}$. The freezing solution was then aspirated, and the well was rinsed once with PBS before the addition of $1 \mathrm{~mL}$ (12well plate) or $300 \mu \mathrm{L}$ (96-well plate) RPMI $1640+10 \%$ NU-SERUM IV or RPMI $1640+9 \%$ FBS. Plates were then returned to the incubator, and growth was monitored throughout the following week.

To assess survival after freezing in multi-well plates, an SV-40-transformed human NB cell line, NB324K $(6,16,17)$, was seeded into 12 - and 96well plates at either $40 \%$ or $90 \%$ confluency. Twenty-four hours later, the culture medium was removed, and the attached cells were overlaid with freezing solution (70\% RPMI 1640, 20\% 